\title{
Uso de Herramientas Clickers en Sesiones Prácticas en los grados de Ingeniería Civil y de HISTORIA
}

\section{Use of “Clickers” in Practical sessions: Civil Engineering and History Degrees}

Javier Estévez Gualda*, Francisco Nicolás Cantero Chinchilla José Antonio Riquelme Cantal, José Luís Sanchidrián Torti

Universidad de Córdoba

* estevez@uco.es

Received: 30/06/2017_Accepted: 15/06/2018

\begin{abstract}
This work studies the impact on students' performance of inserting student-response-systems in practical lessons at the University of Córdoba. Innovative methodologies based on student-response-systems has been developed to be implemented during practical lessons in higher education. A pretest-posttest design is developed and presented in this work to assess the evolution in performance by the students of third course in Civil Engineering Grade at the University of Córdoba during a single course. Finally, motivational questionnaire towards the use of the innovative methodologies and clikers are filled by the students belonging to both Civil Engineering and History grades. The results of this pilot experience show a positive impact on the students' performance by a rapid feedback, in which a conceptual reminder at the beginning of the practical lessons allow to boost the enhancement. The motivational questionnaires described an overall positive students' awareness of the use of the methodologies and clickers. Both motivation and engagement are boosted by the application of the conceptual reminder at the beginning of the practical lessons and, therefore, its consideration is recommended.
\end{abstract}

Keywords: Personal response system; Higher education; Performance; Motivation

\section{Resumen}

Este trabajo presenta un estudio sobre el rendimiento y la motivación de los estudiantes de grado en la Universidad de Córdoba a través de la implementación de clickers durante las sesiones de prácticas. Nuevas metodologías a aplicar durante las sesiones prácticas en ingeniería civil basadas en el uso de clickers son desarrolladas en este trabajo. Para medir el impacto de estas nuevas metodologías, se elabora y se presenta un diseño pretestpostest que se aplicó a los estudiantes de tercer curso del grado en ingeniería civil en la Universidad de Córdoba. Finalmente, los estudiantes de tercer curso del grado de Ingeniería civil y los procedentes del primer curso del grado en Historia completaron cuestionarios de satisfacción para medir el grado de motivación con respecto al uso de las nuevas metodologías y clickers en las sesiones prácticas. Los resultados de esta experiencia piloto demuestran una mejoría en el rendimiento motivado por un feedback casi instantáneo, donde un recordatorio conceptual al comienzo de las sesiones juega un papel fundamental. La motivación de los estudiantes hacia el uso de clickers en las sesiones prácticas es elevada y notable. Tanto la motivación como la atención son potenciadas por el uso del recordatorio conceptual al comienzo de las sesiones prácticas y, por tanto, se recomienda su aplicación.

Palabras clave: Clicker; Educación superior; Rendimiento; Motivación

\section{INTRODUCCIÓN}

El Espacio Europeo de Educación Superior (EEES), con la implantación de los nuevos grados, ha cambiado el paradigma docente. En este sentido, el EEES promueve una implicación del alumnado más activa en clase. Las metodologías docentes basadas en clases magistrales apoyadas en el uso exclusivo de pizarra o presentaciones, por tanto, chocan fuertemente con las directrices marcadas en el EEES. En general, el alumnado espera una metodología más activa y participativa por parte del profesorado, lo que a su vez redunda en un aumento del grado de atención y participación (Wang, 2015). El uso de metodologías expositivas a lo largo de toda la sesión consigue una irrevocable distracción del propio alumnado haciendo un uso no-didáctico de las nuevas tecnologías. En este sentido, la prohibición del uso de aparatos electrónicos (tablets, smartphones y computadoras personales) es difícilmente justificable y poco productiva, ya que en la mayoría de los casos puede derivar en frustración. Por esta razón, es necesario que los docentes integren el uso de nuevas tecnologías en su metodología. Así, el uso de TIC que conjuguen el trabajo individual y colectivo a través de Smartphone y video presentaciones podría ayudar a construir nuevas metodologías docentes que mejoren el rendimiento académico del alumnado, así como su motivación (Roush \& Song, 2013).

Entre la gran variedad de herramientas TIC al alcance de los docentes en la actualidad, los Clickers o "Classroom Response Systems" han irrumpido con fuerza en el EEES. Estas herramientas son fáciles de implementar y aportan grandes beneficios al aprendizaje, así como a la motivación del alumnado (Kay \& LeSage, 2009a; Kay \& LeSage, 2009b; Sun, 2014). El uso de clickers, tales como Kahoot! o Socrative, permiten dinamizar las metodologías docentes. Además, éstos implican el uso de presentaciones interactivas altamente atractivas y Smartphone/Computadora/Tablet para ejercer dicha interacción alumno-contenido en tiempo real (Caldwell, 2007). Asimismo, el uso de clickers genera retroalimentación (feedback) a los 
alumnos facilitando la adquisición de competencias y mejorando el rendimiento académico posterior (Han \& Finkelstein, 2013). El feedback generado mediante el uso de nuevas tecnologías podría llegar a ser más efectivo que la retroalimentación generada por procesos de autoevaluación basados en tests escritos o respondidos electrónicamente mediante plataformas de ayuda a la docencia.

La mayoría de los grados ofertados por la Universidad de Córdoba usualmente orientan las asignaturas para la consecución de competencias a través de altas cargas lectivas basadas en prácticas. Así, mediante la resolución de casos prácticos, el alumnado habitualmente encuentra el sentido último de la asignatura y, por tanto, termina asimilando competencias genéricas o específicas (Ebner \& Holzinger 2007). Sin embargo, el tiempo que transcurre entre las sesiones magistrales teóricas y la aplicación del contenido en sesiones prácticas puede suponer un problema añadido a la comprensión de la materia. Debido a la elevada cantidad de trabajo autónomo, así como a las numerosas asignaturas que cursan nuestros futuros graduados en los nuevos planes de estudios (Plan Bolonia), resulta ciertamente complicado que la mayoría de ellos retenga el contenido antes de ser aplicado en las sesiones prácticas. Desde experiencias con alumnado de años anteriores, este problema aún queda presente. En consecuencia, es importante estudiar el refuerzo de sesiones prácticas a través de metodologías innovadoras con el uso de clickers.

Este trabajo estudia el impacto del uso de Kahoot! en el rendimiento académico y la motivación de estudiantes de grado. El grupo de estudio está constituido por los alumnos matriculados en las asignaturas de Ingeniería Hidráulica Aplicada a Sistemas de Distribución (grado de Ingeniería Civil, Escuela Politécnica Superior de Belmez) y de Sociedades Prehistóricas y sus Entornos (grado de Historia). En primer lugar, se ha realizado un diseño cuasi-experimental pretest postest. En segundo lugar, se han elaborado nuevas metodologías para clases prácticas que ha permitido la aplicación de Kahoot!. Por último, se han aplicado las metodologías, analizados los datos recabados y se han evaluado los resultados en términos de rendimiento, feedback generado y motivación.

\section{OBjETIVOS}

El propósito de este trabajo es evaluar los efectos sobre el rendimiento de los estudiantes de la aplicación de metodologías basadas en el uso de Clickers en sesiones prácticas en dos grados de la Universidad de Córdoba. Este objetivo principal se divide en varios objetivos secundarios: 1) elaborar e implementar metodologías basadas en el uso de Kahoot!, 2) analizar el rendimiento académico tras la aplicación de la metodología propuesta, 3) evaluar la efectividad del feedback generado por las metodologías, y 4) conocer la motivación del alumnado sobre la aplicación de metodologías basadas en Clickers.

\section{METODOLOGíA}

La metodología de este trabajo se ha desarrollado en tres fases consecutivas, de dos semanas de duración cada una, las cuales se detallan a continuación.

\subsection{PRIMERA FASE}

Durante las semanas 1 y 2 se aplicó un diseño cuasiexperimental pretest postest con grupo control. Al comienzo de la semana 1 se aplicó un pretest a ambos grupos sobre los conceptos a trabajar en esta fase. Dichos conceptos fueron explicados previamente en las sesiones teóricas. Durante el estudio, el grupo control siguió una metodología expositiva basada en la resolución exclusiva de casos prácticos. Mientras, el grupo experimental desarrolló la primera metodología integrando Kahoot! para la generación de feedback. Concluidas las dos semanas de la primera fase se aplicó un postest a los dos grupos para evaluar el grado de conocimiento de los conceptos trabajados. Para que la comparación resulte efectiva, ambos tests (pretest y postest) versaron sobre el mismo contenido, variando la redacción de las preguntas para evitar respuestas memorísticas.

La primera metodología aplicada al grupo experimental se basó en un recordatorio de conceptos mínimos al comienzo de la sesión práctica y en la aplicación de Kahoot! una vez terminado el trabajo eminentemente práctico (Figura 1). El recordatorio de conceptos mínimos busca reforzar la memoria de los alumnos con respecto a contenidos ya explicados en sesiones teóricas y que puedan quedar alejados en el tiempo. La duración de las sesiones prácticas en la asignatura Ingeniería Hidráulica Aplicada a Sistemas de Distribución es de 2 horas, por lo que la duración aproximada de las actividades en la metodología será: 5\% del tiempo al comienzo de la sesión para recordatorio de conceptos a trabajar (6 min), 80\% del tiempo para el trabajo práctico (96 min) y 15\% del tiempo en la parte final para la aplicación de Kahoot! (18 min). 


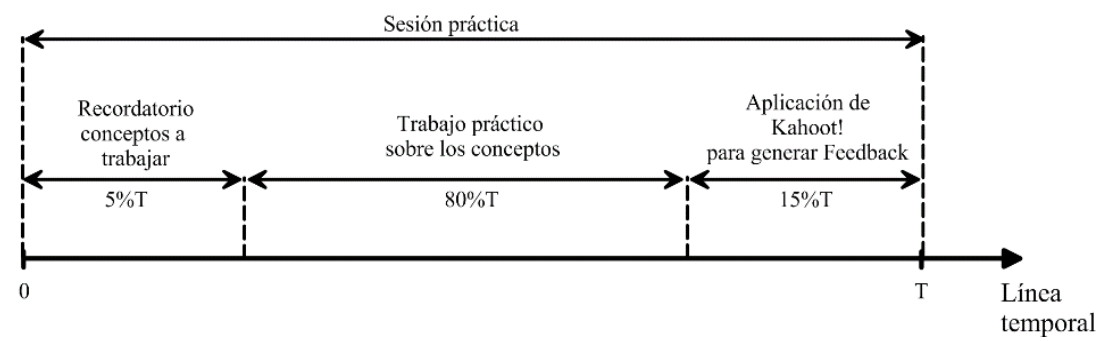

1. Primera metodología propuesta para las sesiones prácticas, donde T es el tiempo de duración de la sesión práctica

\subsection{SEGUNDA FASE}

Durante las semanas 3 y 4 se llevó a cabo el segundo estudio. Se optó también por un diseño cuasiexperimental pretest postest con grupo control. Para garantizar la equidad con los alumnos, los grupos anteriormente definidos como control y experimental invirtieron su rol. Así, el grupo control durante la primera fase pasó a ser el grupo experimental en la segunda fase y viceversa. Al comienzo de la semana 3 se aplicó un pretest sobre los conceptos a trabajar en la segunda fase y que previamente fueron explicados en las sesiones teóricas. En el grupo experimental se desarrolló una segunda metodología con uso de Kahoot!, mientras que el grupo control siguió la metodología expositiva usada en la primera fase. Concluidas las semanas 3 y 4 se aplicó un postest para conocer la evolución del conocimiento de los grupos con respecto a los conceptos trabajados.

La segunda metodología consistió en suprimir el recordatorio de conceptos mínimos al comienzo de la sesión práctica (Figura 2). Así, la comparativa de resultados obtenidos en las fases primera y segunda permitió evaluar la efectividad del recordatorio de conceptos mínimos en las sesiones prácticas. Sobre las 2 horas de duración de la sesión, en esta segunda metodología se estima que el $85 \%$ del tiempo dedicado al trabajo práctico (102 min) mientras que el 15\% del tiempo final de la sesión queda reservado para la aplicación del test Kahoot! (18 min).

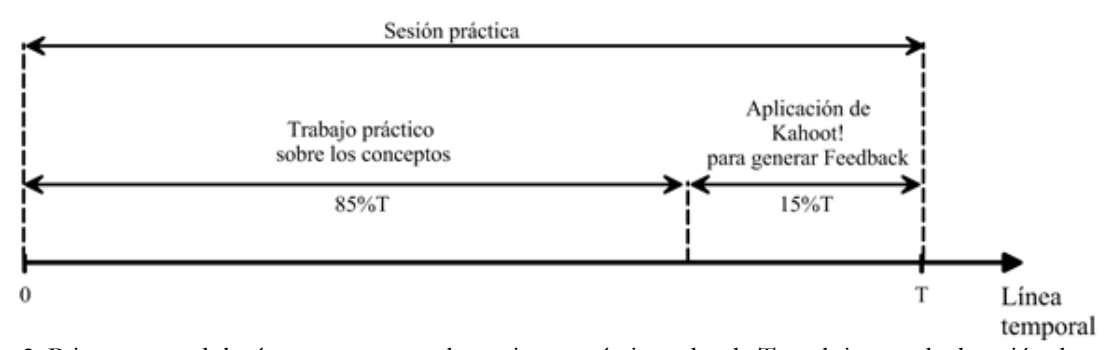

2. Primera metodología propuesta para las sesiones prácticas, donde T es el tiempo de duración de

$$
\text { la sesión práctica }
$$

\subsection{TERCERA FASE}

El último estudio se desarrolló durante las semanas 5 y 6 . Buscando evitar tratos injustos de favor para con alguno de los grupos involucrados en el proyecto, se aplicó a ambos grupos la primera metodología en ésta tercera fase (Figura 1). Así, el test Kahoot! aunó los tests utilizados en las fases primera y segunda. Esto permitió ofrecer el mismo feedback a todos los alumnos de la asignatura, así como estudiar la evolución del conocimiento sobre los conceptos ya trabajados. Al finalizar la semana 6 se aplicó un test de motivación con respecto al uso de las nuevas metodologías. Para ello se eligió el test validado y redactado en inglés propuesto por Kay \& LeSage (2009b). La aplicación de esta tercera fase se extendió del mismo modo a los alumnos de Sociedades Prehistóricas y sus Entornos (grado de Historia).

Los tests de Kahoot! se elaboraron online y de manera gratuita usando https://getkahoot.com/. Posteriormente, los alumnos respondieron dichos tests en clase mediante https://kahoot.it/. Esta última web permite la interacción a tiempo real de los alumnos con el contenido de los tests a través de dispositivos electrónicos tipo Smartphone/Computadora/Tablet.

\section{DESCRIPCIÓN}

Antes del comienzo de las clases prácticas en las cuales se aplicaron las nuevas metodologías, se pasaron los pretest a los estudiantes. Siendo así, el tiempo previsto para la lección práctica no fue reducido. Sin embargo, se requirió una buena disposición de los alumnos a asistir unos minutos antes de la hora prevista a la clase. Una vez concluido el tiempo para contestar el prestest, las lecciones prácticas se desarrollaron dentro del marco de la aplicación de las nuevas metodologías 
(Figuras 1 y 2). Al concluir las lecciones prácticas, un nuevo periodo extra de tiempo se utilizó para pasar los postest a fin de medir el feedback instantáneo generado por las metodologías.

Los prestests y postests son cuestionarios sobre los contenidos de la lección práctica en concreto en los que se estén aplicando, formados por 5 o 6 preguntas de elección múltiple. Para realizar un análisis comparativo entre ambos, los postests reproducen las pregutnas recogidas en los pretests, sin embargo, redactándolas de manera diferente para evitar respuestas memorísticas.

4.1 CRONOGRAMA DE ACTIVIDADES

El cronograma de actividades desarrolladas en este trabajo divididas por fases se detalla en la Figura 3.

\begin{tabular}{|c|c|c|c|}
\hline Fase & Semanas & Grupo 1 & Grupo 2 \\
\hline \multirow{2}{*}{1} & 1 & \multirow{2}{*}{$\begin{array}{c}\text { Grupo control } \\
\text { Metodología expositiva }\end{array}$} & \multirow{2}{*}{$\begin{array}{c}\text { Grupo experimental } \\
\text { Metodología primera con Kahoot! }\end{array}$} \\
\hline & 2 & & \\
\hline \multirow{2}{*}{2} & 3 & \multirow{2}{*}{$\begin{array}{l}\text { Grupo experimental } \\
\text { Metodología segunda con Kahoot! }\end{array}$} & \multirow{2}{*}{$\begin{array}{c}\text { Grupo control } \\
\text { Metodología expositiva }\end{array}$} \\
\hline & 4 & & \\
\hline \multirow{2}{*}{3} & 5 & \multirow{2}{*}{\multicolumn{2}{|c|}{$\begin{array}{l}\text { Grupo experimental } \\
\text { Metodología primera con Kahoot! global }\end{array}$}} \\
\hline & 6 & & \\
\hline
\end{tabular}

3. Cronograma de actividades desarrolladas en el trabajo.

\section{RESULTADOS OBTENIDOS}

Los resultados obtenidos tras comparar los pretests y postests en las diferentes aplicaciones de las metodologías propuestas han demostrado que existe una mejora del rendimiento de los alumnos del grupo experimental con respecto al grupo control. Concretamente, mediante la aplicación de la primera metodología, implementando el recuerdo conceptual al comiendo de la clase, la mejora del rendimiento fue mayor, pasando incluso de un suspenso a un notable en promedio (Tabla 1).

\begin{tabular}{|c|c|c|}
\hline Grupo & $\begin{array}{l}\text { Pretest primera } \\
\text { metodología }\end{array}$ & $\begin{array}{l}\text { Postest primera } \\
\text { metodología }\end{array}$ \\
\hline \multirow{2}{*}{$\begin{array}{c}\text { Experimental } \\
\text { Control }\end{array}$} & 4.7 & 7.3 \\
\hline & 5.4 & 6.9 \\
\hline
\end{tabular}

Tabla 1. Resultados de rendimiento tras la primera metodología.

Por el contrario, la mejora del rendimiento de los alumnos durante la aplicación de la segunda metodología fue menor (Tabla 2).

\begin{tabular}{|c|c|c|}
\hline Grupo & $\begin{array}{l}\text { Pretest segunda } \\
\text { metodología }\end{array}$ & $\begin{array}{c}\text { Postest segunda } \\
\text { metodología }\end{array}$ \\
\hline \multirow{2}{*}{$\begin{array}{c}\text { Experimental } \\
\text { Control }\end{array}$} & 7.2 & 8.4 \\
\hline & 4.8 & 8.2 \\
\hline
\end{tabular}

Tabla 2. Resultados de rendimiento tras la segunda metodología.

La motivación de los estudiantes de los grados de Ingeniería civil e Historia de la Universidad de Córdoba frente al uso de Kahoot! durante las sesiones prácticas fue en general satisfactoria (Tabla 3). En la Tabla 3 se muestra la media de la evaluación de los ítems en el cuestionario de satisfacción de Roush \& Song (2013). Los ítems se dividieron a través de una escala Likert, donde los valores numéricos del 1 al 6 fueron asignados a "fuertemente en acuerdo", "en acuerdo", "neutral", "en desacuerdo", "altamente en desacuerdo" y "no sabe no contesta" respectivamente.

\begin{tabular}{|c|c|}
\hline Item & Evaluación \\
\hline I feel more engaged during class because we use Clickers. & 2,1 \\
\hline $\begin{array}{l}\text { Because we use Clickers, I have a greater sense of participation in } \\
\text { the class. }\end{array}$ & 2,1 \\
\hline $\begin{array}{c}\text { Using Clickers hightens my interest in whatever else we do during } \\
\text { class. }\end{array}$ & 2,2 \\
\hline I wish we spent less time using Clickers. & 3,2 \\
\hline Time spent using Clickers is time well spent. & 2,1 \\
\hline $\begin{array}{l}\text { I have thought about not answering or actually did not answer a } \\
\text { Clicers question because I was afraid someone near me would see how I } \\
\text { answered. }\end{array}$ & 3,3 \\
\hline
\end{tabular}


As I use the Clickers, I am concerned about other students seeing how I answer.

Using Clickers makes me more attentive during lecture or other class activities.

Using clickers helps me focus on the subject matter during class. Using the Clickers technology is easy.

I have no problems using the Clickers technology.

Using the Clickers technology is pretty hard.

Using Clickers boosts my enthusiasm for studying the material we learn in this class.

Using Clickers makes me more motivated to learn in this class.

If we didn't use Clickers, I would be less interested in the topics we cover in this class.

Using Clickers makes me more likely to review my notes prior to class.

Using Clickers encourages me to do readings prior to class.

Because we use Clickers, I prepare for class more than I would otherwise.

Using thee Clickers is fun

It is exciting to answer questions using Clickers technology.

I enjoy using the Clickers technology.

I do not like using clickers.

I have had a good experience with Clickers.

Because Clickers is used, I attend class more regularly than I would otherwise.

Using Clickers increases my likelihood of attending class. Using clickers motivatets me to attend class.

Because of Clickers, I have a better idea of what to expect on exams, quizzes, or assignments.

I believe that I know more about what will be emphasized on exams, quizzes, of assighnments because of Clickers.

Using clickers gives me a preview of what I will need to know for exams, quizzes, or assignments.

Because of Clickers, it is easier for me to tell whether I am mastering class materials.

Because of Clickers, I am more certain about how I am performing in the class.

Using Clickers helps me more easily determine how well I am doing in the class.

My knowledge of class material is improved by using Clickers.

I understand more in this class because we use Clickers.

Clickers help me learn class material better.

Clickers technology is interfering with my getting a good grade.

Because we are using Clickers, I expect to get a lower grade than I

Using Clickers is negatively impacting my grade. would otherwise.

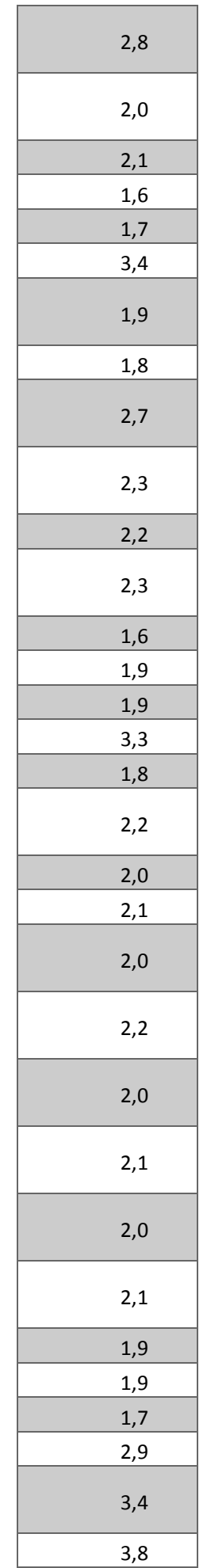

Tabla 3. Resultados de motivación de los estudiantes tras completar el cuestionario de Roush \& Song (2013)

\section{UTILIDAD/ANÁLISIS}

La aplicación de las nuevas metodologías ha permitido mejorar los resultados de rendimiento de los alumnos a través de las lecciones prácticas. El impacto del feedback instantáneo producido por los cuestionarios Kahoot! fueron, sin duda, en gran medida responsables de esta mejora. Resulta conveniente reseñar que durante los test de Kahoot! al final de las sesiones se abrió un foro de debate sobre las respuestas correctas e incorrectas de manera instantánea tras contestar cada una de ellas, dando lugar a un fuerte feedback. Además, mediante la implantación del recordatorio de conceptos mínimos al comienzo de 
la clase, los resultados de rendimiento mejoraron aún más. Esto se puede explicar desde el punto de vista del refuerzo de los conceptos que se trabajan en las sesiones prácticas.

El uso de la herramienta Kahoot! por parte del docente no requiere demasiado tiempo de atención, pudiendo preparar un cuestionario de 10 preguntas de respuesta múltiple en 30 minutos con conocimientos previos de ofimática. Además, el análisis cualitativo de la aplicación de estas metodologías basadas en Clickers demuestra un mayor grado de dinamización en las lecciones prácticas, que favorece la integración y la atención de los estudiantes.

Los resultados de motivación de los estudiantes frente al uso de Kahoot! en clases prácticas fue, por lo general, muy satisfactorio. En especial reseñaron que encuentran fácil su utilización y que les motiva y ayuda en su rendimiento durante las clases.

\section{CONCLUSIONES/DISCUSIÓN}

El presente trabajo pone de manifiesto la constatación de una mejora en el rendimiento académico y la motivación focalizado en lecciones prácticas en los grados de Ingeniería Civil e Historia la Universidad de Córdoba mediante el uso de metodologías basadas en la integración de sistemas Clickers. La implementación de nuevas metodologías en lecciones prácticas frente a las metodologías clásicas aumenta el grado de atención de los alumnos, impactando favorablemente en su rendimiento a través de un fuerte feedback. Entre las metodologías innovativas propuestas, aquellas que integran recordatorios de conceptos mínimos al comienzo de las lecciones demuestran una mejora superior del rendimiento de los alumnos y, por tanto, se recomienda su implementación.

\section{BIBLIOGRAFÍA}

Caldwell, J. E. "Clickers in the large classroom: Current research and best-practice tips", CBE-Life Sciences Education 2007/6(1), p. $9-20$

Ebner, M., Holzinger, A. "Successful implementation of user-centered game based learning in higher education: An example from civil engineering", Computers \& education 2007/49(3), p. 873-890

Han, J. H., Finkelstein, A. "Understanding the effects of professors' pedagogical development with Clicker Assessment and Feedback technologies and the impact on students' engagement and learning in higher education", Computers \& Education 2013/65, p. 64-76

Kay, R. H., LeSage, A. "Examining the benefits and challenges of using audience response systems: A review of the literature", Computers \& Education 2009a/53(3), p. 819-827

Kay, R. H., LeSage, A. "A strategic assessment of audience response systems used in higher education", Australasian Journal of Educational Technology 2009b/25(2), p. 235-249

Roush, C., Song, L. "The impact of using clickers technology on classroom instruction: students' and teachers' perspectives", The Canadian Journal of Action Research 2013/14(1), p. 21-37

Sun, J. C. Y. "Influence of polling technologies on student engagement: An analysis of student motivation, academic performance, and brainwave data", Computers \& Education 2014/72, p. 80-89

Wang, A. I. "The wear out effect of a game-based student response system", Computers \& Education 201582, p. 217-227 